\title{
Computer-Aided Surgery Planning for Lower Limb Osteotomy
}

\author{
Anja Perlich ${ }^{1}$, Bernhard Preim ${ }^{1}$, Marie de La Simone ${ }^{2}$, Christophe Gomes ${ }^{2}$, \\ Eric Stindel ${ }^{3}$, Ana Presedo ${ }^{4}$ \\ ${ }^{1}$ Institut für Simulation und Grafik, Fakultät für Informatik, OVGU Magdeburg \\ ${ }^{2}$ EOS imaging, Paris \\ ${ }^{3}$ Centre Hospitalier Universitaire, Brest \\ ${ }^{4}$ Hôpital Robert Debré, Paris \\ aperlich@stud.hs-heilbronn.de
}

\begin{abstract}
Osteotomies around the knee address lower limb deformities that affect the leg posture and especially the knee joint. Knee arthritis is a very common age-related joint disease that can be treated with osteotomy, as an alternative to knee replacement by implants. The preoperative planning process is demanding and significantly determines the surgical out-come. The choice of the osteotomy type and the determination of parameters, like the correction angle, are crucial for long-term success. We present a software tool for preoperative 3D planning of femoral and tibial opening, closing and derotation osteotomies, thus a particularly wide range of interventions. Our tool is based on patient specific $3 \mathrm{D}$ bone models and allows the user to position cutting planes and to simulate cut and alignment of the bone.
\end{abstract}

\section{Introduction}

Lower limb osteotomies correct different forms of malalignment. A common disease, attended by varus (bowlegs) or valgus (knock-knees) deformity, is knee osteoarthritis. Those deformities are addressed by opening or closing osteotomy which try to establish a better alignment by passing the load bearing leg axis through hip, knee and ankle joint. Another type of deformity, the inner torsion of the bone, may result in disturbance in gait and can be addressed by derotation osteotomy (Fig. 1). In order to exactly define the characteristic of the osteotomy, angles between bone and limb axes are measured. Thus the deformity can be located and the anatomical parameter to be corrected can be determined. Following, the osteotomy mode, bone, level and orientation of the cut are determined. An important issue is the correction angle, ie. how many degrees to open, close or rotate the bone. These parameters are crucial for the good out-come of the surgery.

The femorotibial mechanical angle (FTMA), measured on a frontal standing radiograph, characterizes the magnitude of varus/valgus and is important in opening and closing osteotomy [1]. In order to assess and correct a rotational malalignment (torsion), angles have to be measured on a projection along the 
bone axis. Computer support can accelerate the calculation and the exactitude of the planning. There are 2D and 3D osteotomy planning tools [2]. Even if the 2D tools can help to evaluate faster and more correctly the patient's situation, there are error sources due to the $2 \mathrm{D}$ projection radiography that misses information which is necessary to globally assess the anatomy and to determine surgery parameters. Ellis et al. [3] developed a 3D surgical planning and guidance system for high tibial osteotomy based on computed tomography data. A drawback is the high irradiation exposure for the patient.

Brainlab developed VectorVision $\AA$ osteotomy, an intra-operative planning and navigation system for high tibial osteotomy [4]. The 3D models of femur and tibia are based on important landmarks and provide information of the patient's lower limb anatomy. Apparently, the used bone model can only represent a rather limited number of patient specific landmarks. This could lead to wrong evaluation if, for instance, existing bone deformities are not visible in the model. The goal of our work was the prototyping of a tool not limited to one bone or osteotomy mode.

\section{Materials and Methods}

We developed a preoperative osteotomy planning system for surgeons, based on patient specific 3D models provided by SterEOS [2]. Our prototype OstEOS was programmed in Matlab and is based on femur and tibia triangle meshes (Fig. 2). We implemented algorithms to cut triangle meshes and to transform the resulting lower part in order to enable simulations of opening, closing and derotation osteotomy. The graphical user interface enables the user to evaluate the lower limb anatomy by exploring the bone models and parameters, to position the cutting plane, and to simulate the cut and alignment of the bone. Various parameters calculated in real-time can be seen while the user simulates

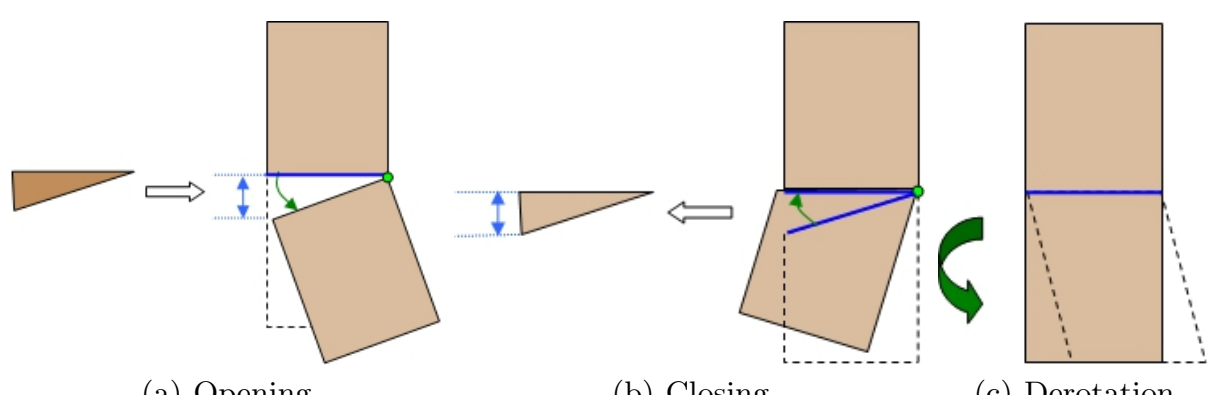

(a) Opening

(b) Closing

(c) Derotation

Fig. 1. Osteotomy modes that were implemented. The bone is cut ones and the lower part is rotated about a certain hinge axis. The resulting gap can be filled with a wedge (a). The bone is cut twice, the bone wedge is removed, and the lower part is fold up to close the gap (b). The bone is cut one time and the lower part is rotated around a bone axis. 
Fig. 2. Proximal end of the femur. A cut result of a closing osteotomy is shown.

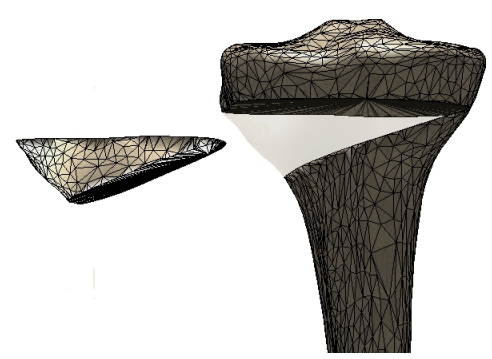

the osteotomy. There are pre- and post-operative bone parameters (length, torsion and angles between bones) and surgery parameters (correction angle, level of the cutting plane and the bone wedge base height) (Fig. 3).

We implemented an algorithm to determine the correction angle given the desired postoperative FTMA angle using trigonometrical laws [2]. In practice, osteotomy planning tools are relevant because surgery parameters can be determined preoperatively. The 3D simulation of the expected osteotomy result helps the surgeon to better understand the effects of the bone alignment. In contrast to the majority of existing 3D tools, OstEOS is not limited to one type of osteotomy, but allows the planning of femoral and tibial opening, closing and derotation osteotomy. It is based on detailed patient specific bone models and gives a reliable representation of the anatomy. By clicking on arrow buttons or by typing the desired correction angle or FTMA in a GUI input field, the user can position the cutting plane(s) and, after the cut, align the lower part.

In the opening and closing mode, the cutting plane(s) can be translated along the $z$ axis, rotated around a hinge axis perpendicular to the frontal plane of the bone frame and it can be fitted to the bone amplitude at a certain level. This is useful to fit the hinge axis on the bone surface. In derotation mode, the cutting plane does not have a hinge axis and can be translated along the $z$ axis.

The GUI consists of two parts (Fig. 3). The right part contains the 3D scene with bone and cutting plane objects. To enable optimal perception, a radiograph-like color scheme is used: the background color is dark and the bones are visualized in a lighter, natural color. The left part contains patient and surgery data as well as buttons for the osteotomy determination and simulation. Thus the effect of user inputs can be seen directly when changing parameter values. Expert talks along with demonstrations of our software helped us to confirm the correctness and appropriateness of the tool during the development.

A final test was made to evaluate the usability of OstEOS. Test persons were two osteotomy practicing surgeons. They were asked to plan and virtually execute three osteotomies using OstEOS, performing one opening, one closing and one derotation on pathologic bone models. Person 1, a surgeon interested in computer support, plans osteotomies manually on 2D radiographs. Person 2, a pediatric surgeon not familiar with software tools for osteotomy planning, does approximate planning and uses rarely planning sketches due to the fact that children's growing is not stable. 


\section{Results}

The time taken for the planning of one surgery by person 1 was 3-4 minutes. He commented that he was able to perform all necessary planning steps, that he would like to show the tool to colleagues and that there were no barriers using OstEOS. Wrong decisions could be easily undone with the help of reset buttons. The approach to the osteotomy simulation and the steps to attain a cut and axis alignment were clear. Person 1 was able to get the answers on his questions; for example where to place the cutting plane, how many degrees to open the bone and which size of wedge to use to fill the resulting gap. The 3D bone representation helped to comprehend the shape of bone and and surgery effects. Person 2 took 5-10 minutes and had been helped when she was stuck. She found the tool very useful but in her opinion some functions were missing, e.g. the possibility to locate the center of deformity (see [5]).

\section{Discussion}

The user test showed that all important basic functionalities exist, but that further functionalities need to be implemented in order to enlarge the spectrum of

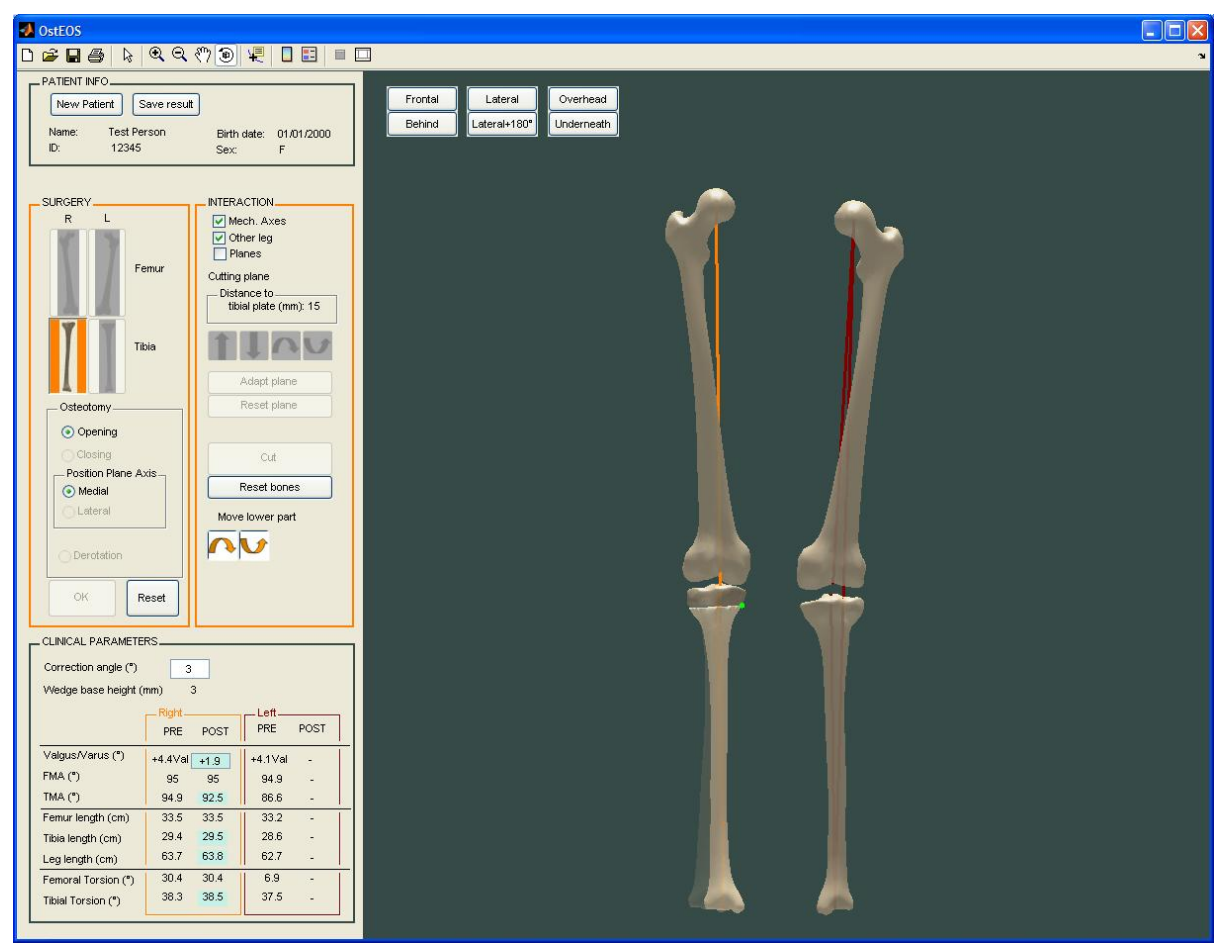

Fig. 3. Graphical user interface of OstEOS. 
supported patient cases, and to allow the planning of more advanced osteotomies. The efficiency is very satisfying since the planning can be done in three minutes, whereas a traditional planning with manual calculations can take up to 15 minutes. The pre and postoperative parameters, updated and displayed in real time, contributed a lot. Both test persons were interested in using a tool like OstEOS in clinical routine work. OstEOS allows the planning of many types of osteotomy and thus stands out from other 3D planning tools which are often limited to high tibial osteotomy (see [6]). Another benefit is the reliable 3D representation of the anatomy by the detailed patient specific bone models.

We conclude that the $3 \mathrm{D}$ aspect enriches the planning in comparison to $2 \mathrm{D}$ tools, but that it holds further challenges. Most notably is the interaction with the $3 \mathrm{D}$ scene that requires practice. Ideally, a planning tool should allow a realistic simulation of all manipulations that can be done during the surgery in the operating block. Future prospects are a better user-interaction with the 3D scene, the automation of the process and to take into account the knee joint force distribution.

\section{References}

1. Pape D, Lorbach O, Steimer O. Analyse der Deformität und präoperative Planung einer knienahen Osteotomie. Arthroskopie. 2007;20:277-90.

2. Perlich A. Computer-aided surgery planning for lower limb osteotomy.; 2010.

3. Ellis RE, Tso CY, Rudan JF, et al. A surgical planning and guidance system for high tibial osteotomy. J Comput Assist Surg. 1999;4(5):264-74.

4. Brainlab. Brainlab VectorVision Osteotomy; 2010.

5. Paley D, Pfeil J. Prinzipien der kniegelenknahen Deformitätenkorrektur. Orthopäde. 2000;29:18-38.

6. Lobenhoffer P, van Heerwaarden R, Staubli A, et al. Osteotomies around the knee: indications, planning, surgical techniques using plate fixators. AO Foundation; 2008. 